\title{
UNA NUEVA VITALIDAD PARA EL FUTURO DE LOS COMITÉS DE ÉTICA ASISTENCIAL
}

\section{Rogelio Altisent, Teresa Fernández-Letamendi, $M^{a}$ Teresa Delgado-Marroquín}

\begin{abstract}
Resumen: Los comités de ética asistencial se encuentran en una fase determinante para su consolidación. Es necesario no obstante mejorar su conocimiento entre los profesionales y la sociedad, así como la accesibilidad y la sintonía con las auténticas necesidades de su entorno asistencial. Para ello es muy conveniente articular procedimientos que den agilidad de respuesta a quienes solicitan asesoramiento, donde la figura de los consultores puede ser de ayuda. La evaluación de la calidad será un aspecto clave de esta etapa, en la que los comités deben demostrar, con indicadores que permitan establecer comparaciones, su eficiencia. Asimismo los centros sanitarios necesitan gerentes con capacidad de liderazgo y sensibilidad para entender la trascendencia de lo que es un comité de ética asistencial y su potencial contribución a la vida del sistema sanitario, lo cual se debe defender proporcionando los recursos necesarios para garantizar su adecuada actividad.
\end{abstract}

Palabras clave: Comités de ética asistencial/ profesionalismo/ mejora de la calidad.

Title: NEW VITALITY FOR THE FUTURE OF CLINICAL ETHICS COMMITTEES.

\begin{abstract}
Clinical ethics committees have reached a decisive stage in their consolidation. It is necessary to improve the knowledge that healthcare professionals and society have of them, and their accessibility and alignment with the needs of the healthcare environment. It is highly desirable that procedures should be managed in such a way as to expedite their response to those requesting advice, where the figure of ethics consultants may be of assistance. Quality assessment with indicators that enable comparisons to be made will be a key aspect at this stage when committees must demonstrate their efficiency. Health centres require managers with leadership skills and the sensitivity to understand the significance of a clinical ethics committee and its potential contribution to the life of the health service, which requires the provision of the necessary resources that guarantee an appropriate level of activity.
\end{abstract}

Keywords: Ethics committes/medical professionalism/ quality improvement.

Artículo recibido: 24 julio 2019; aceptado: 11 noviembre 2019.

En la historia de las instituciones, que perviven en el tiempo, se suelen repetir una serie de etapas. La primera es un arranque carismático protagonizado por personas notables, que dan respuesta a una necesidad real mediante la organización de una estructura funcional que es necesariamente muy dependiente de las cualidades de sus líderes fundadores. A continuación, viene la etapa de consolidación institucional, mediante la creación de normas y reglamentos que dan estabilidad, más allá de los voluntarismos, con el fin de que las funciones y las tareas institucionales trasciendan a las personas, permitiendo 
el sucesivo relevo de los responsables. Por último, si todo aquello merece la pena, viene la fase de la continuidad, que generalmente necesita nuevos impulsos para evaluar la actividad y garantizar la sostenibilidad, siendo necesario realizar ajustes y reformas fundamentadas en la experiencia del recorrido previo.

En esta reflexión defenderemos la tesis de que, en España, los comités de ética asistencial en las instituciones sanitarias (CEA) han pasado las dos primeras fases. Nos encontramos ahora en la tercera etapa de continuidad, donde está en juego su futuro, que será de decadencia o de florecimiento, en dependencia de una serie de elementos que discutiremos, a continuación, con el ánimo de contribuir a promover una nueva vitalidad.

\section{APUNTES HISTÓRICOS}

Decía Claudio Magris que la vida hay que vivirla mirando hacia delante, pero solo se puede entender mirando hacia atrás. Recordemos que, desde mediados del siglo $X X$, hemos asistido a un creciente interés por la bioética en todo el mundo. Entre otros motivos, se ha producido una noble reacción contra la deriva de una medicina que, fascinada por la tecnología, amenaza con perder de vista que en el centro de su atención hay seres humanos dotados de dignidad. En la memoria histórica quedan las iniquidades de la investigación que hizo la medicina del nazismo y el uso político de los soviéticos; pero también, y más recientemente, en países supuestamente respetables se cometen abusos en nombre de la medicina y de la ciencia que no se pueden considerar totalmente erradicados.

El escenario sanitario de estos últimos 50 años se ha caracterizado por tres cambios trascendentales, de los que muchos hemos sido testigos de primera mano: los espectaculares avances científicos, la eclosión de la autonomía del paciente y las modernas organizaciones sociales de prestación sanitaria. Este panorama ha generado nuevas cuestiones éticas que nunca antes se habían planteado. Son nuevas preguntas que requieren nuevas respuestas. Hace 100 años no se planteaban problemas éticos ante: la retirada de un respirador en una 
unidad de cuidados intensivos, ni se discutía sobre si es moralmente aceptable comprar un órgano para trasplantar, o si los fumadores debían pasar a la cola en la lista de espera de cirugía coronaria; sencillamente porque no había unidades de cuidados intensivos, ni trasplantes de órganos, ni cirugía cardiaca.

Este es el paisaje donde hemos visto nacer y crecer los comités de ética para ayudar en la toma de decisiones éticas difíciles.

La prehistoria de los CEA se suele situar hacia 1960, cuando se puso en marcha la primera unidad de diálisis en Seattle (USA). Se constituyó entonces un comité para ayudar a decidir sobre el acceso de pacientes a las máquinas de diálisis, que en aquellos primeros momentos eran insuficientes para atender al número de enfermos con insuficiencia renal que eran candidatos a su uso. La primicia fue, además, que algunos miembros de este comité no eran médicos, porque se entendió que los criterios meramente técnicos no eran suficientes para decidir quién debía tener prioridad en el acceso a la diálisis. Se sintió la necesidad de tener diferentes perspectivas para acertar en la toma de decisiones, es decir, contar con otras miradas como, por ejemplo, la de los cuidadores que conviven con los pacientes, siendo capaces de conocer sus preferencias y valorar lo que para ellos era auténtica calidad de vida'.

Los manuales de historia de la bioética también acostumbran a citar cuando, en 1968, se creó un comité de la Facultad de Medicina de Harvard para establecer el criterio de la muerte cerebral y definir cuándo un paciente ha muerto, afinando el momento en que ya se le puede hacer una extracción del órgano de manera que sea útil para el trasplante. Otro hito reiteradamente mencionado aconteció en 1976 en los EE.UU, el caso de Karen Ann Quinlan, una joven en coma profundo por ingesta de alcohol y barbitúricos, cuyos padres solicitaron retirar la ventilación asistida. El Tribunal Supremo de Nueva Jersey dictaminó que autorizaría la demanda si, tras la constitución de un comité de ética en el hospital donde estaba ingresada la paciente, éste confirmaba la irreversibilidad del coma. Casos como éstos son tan solo una muestra de las experiencias más citadas que ayudan a entender la prehistoria de los CEA. 
A partir de los años ochenta, del siglo $X X$, es cuando se produce una espectacular extensión de comités de ética en los EE.UU.: pasando del $1 \%$ de los hospitales en 1982 al $60 \%$ en 1987 . Hacia el año 2000 se estimaba que el 93\% de los hospitales tenia algún tipo de comité de ética, y hoy ya se encuentran prácticamente en el $100 \%$.

Los primeros comités de ética asistencial en hospitales públicos españoles datan del año 1993. El impulso de las primeras promociones del Máster de Bioética de la Universidad Complutense de Madrid dirigido por el Profesor Diego Gracia, logrando que en pocos años más de 30 hospitales tuvieran un CEA en funcionamiento. Aunque para hacer justicia histórica también hay que mencionar la experiencia pionera liderada por Francesc Abel en el Hospital de Sant Joan de Deu de Barcelona, que en 1974 marcó un hito en la historia mundial de los CEA.

En 1995, el Ministerio de Sanidad a través del entonces INSALUD (ente responsable de la asistencia sanitaria del Sistema Nacional de Salud español hasta su transferencia a las comunidades autónomas) dictó una normativa en España que regulaba la constitución de los CEA, que se fueron extendiendo por todo el territorio nacional. Estas directivas se dirigían sobre todo a los hospitales, aunque ya contemplaba la posible integración de la atención primaria. En Cataluña y en el País Vasco, donde ya se habían realizado las transferencias sanitarias con anterioridad, se regularon los CEA en 1993 y 1995 respectivamente $^{2}$. A partir del año 2000 las comunidades autónomas -a excepción de Navarra y Extremadura- han ido creando su propia normativa que regulan la constitución y acreditación de los CEA.

En estos momentos se acepta que una institución sanitaria no tiene su organigrama completo si no tiene un CEA de referencia operativo, donde los profesionales, los usuarios y sus familiares puedan acudir a pedir asesoramiento. Sin embargo, sigue existiendo un gran desconocimiento sobre su existencia y funciones, aunque ciertamente se ha avanzado notablemente en los últimos 10 
años, en buena medida porque los estudiantes de medicina reciben enseñanza reglada de ética donde se suele hablar con naturalidad de los CEA.

\section{¿QUÉ PUEDEN APORTAN LOS CEA?}

EI CEA debe ser un espacio para la búsqueda del conocimiento moral, un lugar donde se cultive la reflexión de la dimensión ética y humana de la asistencia sanitaria.

Hemos tomado conciencia de que la evidencia científica es necesaria pero no suficiente para prestar una buena asistencia. Por tanto, no nos basta con ser unos estupendos científicos conocedores de las últimas novedades que se han publicado; tampoco es suficiente con ser unos virtuosos de la técnica más avanzada, ya sea para operar un tumor cerebral, ya sea para tratar una ulcera de decúbito. En nuestros centros y en nuestras consultas también necesitamos crear un clima de confianza, de respeto a la autonomía y a la vida; que en nuestros centros se garantice la confidencialidad, la coordinación de los equipos, el compromiso con el paciente, la cultura de servicio, la honestidad, la transparencia, la equidad, la adecuada gestión de los conflictos de interés, etc. En definitiva, se trata de cultivar una serie de valores que parafraseando a Popper 'no se dejan atrapar por el método experimental', y que son precisamente el objeto de estudio de la ética ${ }^{3}$.

Buscamos el conocimiento moral mediante el razonamiento ético y la deliberación. A diferencia del conocimiento científico que alcanzamos mediante el método experimental, el ensayo y la refutación. Este matiz es importante porque no se puede perder de vista que mientras el conocimiento científico busca la certeza, a través del conocimiento moral intentamos alcanzar el juicio prudencial. Son dos perspectivas diferentes pero sinérgicas, que deben ir de la mano para lograr una auténtica calidad asistencial.

El CEA es un lugar para cultivar la deliberación y la prudencia. Recientemente se ha difundido un reportaje sobre el templo de la Sagrada Familia de Barcelona realizado con un dron (un vehículo aéreo no 
tripulado), dotado de ocho rotores. Éste ha permitido ver ángulos y perspectivas que nunca antes se habían captado de la célebre obra de Gaudí, inalcanzables en una visita guiada convencional. Esta pluralidad de miradas, con una perspectiva interdisciplinar, es una de las principales aportaciones de un CEA, al ofrecer una riqueza de visiones que difícilmente podrá alcanzar un profesional de manera individual.

Sin embargo, sería desatinado insinuar que la ética ha llegado a la medicina con la creación de los comités, porque es bien conocido que no hay una profesión más impregnada de valores morales desde su nacimiento. No podemos olvidar que el impulso y el motor del comportamiento está en la ética de cada persona, esto es lo que hace que las cosas funcionen. El principal lugar de deliberación ha de estar en la cabecera del paciente, en la sesión del equipo o en el despacho donde se informa a las familias. No pretendemos decir que los comités sean la fuente de la moralidad, ni la última palabra que se debe acatar. No obstante, acumulamos ya abundante experiencia de cómo los CEA pueden ser un lugar de apoyo y de promoción de la dimensión humana de la asistencia sanitaria. Por ello, un comité ha de ser un grupo que inspire confianza y donde, quienes acuden a consultar, se sientan acogidos y nadie piense que va a ser juzgado o humillado.

También podemos avanzar que el CEA será en algún momento como la conciencia de la institución: una caja de resonancia de las cuestiones éticas que se plantean a la cabecera del paciente o en el centro sanitario de manera general. Un lugar donde se generen iniciativas de formación reposadas y maduradas, pero también con agilidad, con reactividad ante los problemas que preocupan. Por ejemplo, sería un buen indicador de vitalidad de un CEA la promoción de un seminario sobre dilemas éticos en la alimentación de pacientes con demencia (una preocupación común de geriatras, neurólogos, internistas, médicos de familia, enfermería, tanto de hospital como de atención primaria).

Podemos aspirar a que el CEA se convierta en: una fuente de motivación que busque contagiar a todos los equipos, servicios y centros de salud, creando 
metástasis, mediante una red de contactos, en cada unidad que colaboren con el comité y que, así, sean el germen para impulsar iniciativas sencillas. Por ejemplo, para conseguir que, en las sesiones clínicas, se analicen aspectos éticos del caso en cuestión, sin necesidad de que hayan planteado especiales dilemas, sencillamente evaluando cómo se ha manejado la información o la confidencialidad.

De todo lo anterior es fácil deducir que trascendemos el ámbito exigido por las leyes. Por ello, en algún momento, se pueden suscitar dudas legales y, para resolverlas, cada comité tiene un miembro con experiencia en derecho sanitario, pero con la idea clara de que no estamos para resolver consultas jurídicas. El fin último de la ética es la excelencia, o dicho con lenguaje llano, hacer las cosas bien, lo mejor posible, lo cual supone una aspiración que trasciende la norma legal que se orienta a garantizar derechos básicos.

\section{¿QUÉ TIPO DE CEA NECESITAMOS?}

Las características que en estos momentos se necesitan en un CEA para que sea una institución útil y no meramente decorativa son en nuestra opinión:

Sintonía. Se necesita que un CEA sintonice con las necesidades reales de los profesionales y los usuarios. En otras palabras, no puede ser un grupo autista ni un cenáculo de ilustrados que se consideran un peldaño por encima de los demás.

Además de atender las consultas de casos complejos, se deben estudiar los temas de mayor prevalencia y de mayor dificultad en la toma de decisiones. Para ello, conviene conocer la epidemiología ética de lo que está ocurriendo en el entorno asistencial de referencia ${ }^{4}$. Con este fin, necesitaremos realizar una tarea de investigación, para identificar cuales son esas cuestiones. Esta función investigadora se encuentra explícitamente mencionada en el Decreto de regulación de comités de Aragón y de Baleares, y se añade a las tres funciones tradicionales (docencia, consulta de casos, elaboración recomendaciones). Esto tiene algunas consecuencias relevantes a tener en cuenta. Por ejemplo, 
necesitamos mejorar la formación en metodología de la investigación, sobre todo cualitativa, porque nos será muy útil para lograr este fin: disponer de un mapa de epidemiología ética.

Este esfuerzo por sintonizar con las necesidades sentidas y reales del escenario asistencial circundante, estimulará el intercambio con áreas asistenciales que desconocen las tareas y funciones del CEA. De este modo se promueven nuevas consultas de casos y actividades de formación en bioética ajustadas a los aspectos que los profesionales consideran de mayor ayuda. Un buen ejemplo de sintonía del CEA con su entorno sería el acercamiento a una residencia de ancianos de la zona para explorar los problemas éticos que se les presentan, con el fin de explicarles la ayuda que el CEA puede aportar.

Accesibilidad. El CEA debe ser accesible a los profesionales, a los usuarios y sus familias cuando sea conveniente realizar una consulta. De un modo amable y sin exceso de formalismos, en un primer momento, aunque las auténticas consultas de casos deban presentarse por escrito, para lo cual a menudo conviene prestar apoyo a través de un "consultor".

Hemos de facilitar el acceso a distancia al CEA: desde la consulta de un centro de salud o desde la planta de un hospital o desde una residencia de ancianos. Esto requiere disponer de vías telemáticas sencillas y de fácil manejo.

Para los equipos directivos no existe un problema de accesibilidad. Es frecuente y normal que en el ámbito de la gestión se planteen cuestiones tributarias de ser estudiadas por un CEA. La experiencia de estas décadas de CEA en España ofrece un amplio abanico de situaciones. En muchos casos la relación es fluida, para lo cual la actitud de colaboración mutua es decisiva. Cada vez se dan menos casos de desconocimiento, aunque todavía hay algunos directivos que piensan que el CEA les puede dar más problemas de los que ya le genera la propia gestión.

Agilidad. Hay que equilibrar la necesaria calma de la deliberación con cierta reactividad a la hora de responder una consulta. Conviene matizar y 
diferenciar el tipo de consultas que se reciben, porque no todas van a necesitar el mismo tratamiento:

a) Consulta exprés: sería una duda puntual que se puede resolver directamente sin necesidad de recurrir a un pleno. Hay casos que se pueden enfocar con una conversación donde, en el fondo, se ayuda a deliberar a quien consulta para que alcance una decisión que se considera razonable y prudente. A veces son cuestiones que ya están resueltas por una norma legal o deontológica, y en ocasiones se pueden encauzar con una orientación bibliográfica.

b) Consulta inmadura o incompleta. Se trata de la consulta de un caso complejo, pero que no está del todo madura en su planteamiento. En estas situaciones resulta muy útil el apoyo de un consultor que ejerza de "hada madrina" ayudando a su presentación, por escrito, para que pase a su estudio en un pleno del CEA.

c) Caso adecuadamente presentado. Elaborado de acuerdo con el protocolo y los requisitos que establece el comité, que deben estar asequibles en la web del CEA. Una vez presentado el caso, el CEA confirmará por escrito la recepción y su aceptación, concretando una estimación del tiempo de su respuesta y comunicando que será analizado en el siguiente pleno. En el caso de una decisión urgente, se convocará la comisión de urgencia, para lo cual es muy conveniente comisionar a un "consultor" que realice las tareas de enlace.

Eficiencia. Es una de las asignaturas pendientes que hemos de aprobar para alcanzar la mayoría de edad de un CEA. Deberemos demostrar que los recursos invertidos (horas de profesionales altamente cualificados) se traducen en un cuidado de la dimensión ética que produce una mejora de la calidad asistencial. No podemos admitir, sin más, que un grupo de personas muy cualificadas y llenas de buena intención, que dedican varias horas al mes en su jornada laboral a un CEA, se auto-justifiquen de manera automática. Al igual que cualquier otra comisión o grupo que trabaja en la institución, el CEA supone una inversión de recursos que debe producir resultados para justificar su existencia. 
Es natural que se necesite un tiempo de gestación y formación, pero siempre hay que tener en el punto de mira la evaluación y la mejora de la calidad del trabajo desarrollado.

Evaluación. La evaluación es un requisito para demostrar la eficiencia. Un primer paso para esta estrategia es cumplir los criterios exigidos para la acreditación. Se trata de un conjunto de garantías básicas en cuanto a la estructura y el funcionamiento, pero también se debe desplegar un sistema de evaluación continuada de la actividad desarrollada plasmada en la memoria anuals.

La Unidad de Acreditación de los CEA debe evaluar la memoria de actividades, con la ayuda de consultores externos, no tanto con una finalidad fiscalizadora como para indicar áreas de mejora. La memoria permitirá al menos realizar una valoración cuantitativa de: reuniones, asistentes, consultas recibidas, informes escritos, documentos de recomendaciones, alertas éticas a la institución, actividades formativas internas y externas con número de asistentes, proyectos de investigación y publicaciones; tiempos de resolución de los procedimientos. Será muy conveniente seleccionar algunos indicadores que servirán además para realizar comparaciones con otros $C E A^{6-8}$.

Es también muy conveniente completar la evaluación cuantitativa con una valoración cualitativa complementaria, mediante encuestas de satisfacción a los propios miembros del CEA, a los profesionales, pacientes o familiares que consultan, y al equipo de dirección del centro.

\section{CUESTIONES ABIERTAS AL DEBATE}

Una reflexión abierta sobre el futuro de los CEA y su vitalidad nos suscita varias cuestiones que son actualmente objeto de debate. Un esbozo:

Escaso conocimiento de los CEA. Tenemos pocos estudios empíricos al respecto, pero todos coinciden en la percepción de que los CEA no se conocen bien. Aunque hemos de reconocer que en los últimos años se está avanzando en este sentido, sigue siendo bajo el conocimiento de su existencia y, aun menor, 
el de sus funciones. Se necesita desarrollar estrategias de difusión que informen con pedagogía, de un modo amable, con un estilo cooperativo para que se genere confianza y cercanía, de tal modo que se haga fácil acudir a consultar o a solicitar colaboración para una sesión.

Sabemos que la educación es una inversión que no tiene un rendimiento inmediato. La formación necesita tiempo para sedimentar, pero también hace falta programación y estrategia. Tiene una especial importancia plantearse en el CEA el modo de llegar a los sectores profesionales más refractarios o escépticos. Sin embargo, la promoción de un comité de reciente constitución se debe hacer progresivamente y midiendo las fuerzas, porque si un CEA joven y entusiasta genera muchas expectativas y luego no las atiende adecuadamente se hace el harakiri.

Se reciben pocas consultas. Esto, que a veces genera frustración en los comités, debe ser adecuadamente interpretado. A menudo existe un sub-registro de consultas a miembros del CEA que se hace de manera informal, pero que se resuelven como lo que hemos denominado consultas exprés, y que no constan en el acta ni en la memoria. Es importante que el comité articule el modo de atender y registrar este tipo de consultas.

Naturalmente, para facilitar la recepción de consultas, hay que mejorar la accesibilidad. Está resultando de gran ayuda desarrollar la figura de los "consultores" 10 pues neutralizan algunos de los motivos que frenan a algunos profesionales a consultar, como puede ser la sobre-exposición ante un grupo numeroso de colegas o el temor a la pérdida de confidencialidad.

Indudablemente, un factor determinante del bajo número de consultas es el déficit de formación en este campo. A veces a los profesionales les cuesta identificar las cuestiones éticas, o las confunden con las legales. La experiencia nos muestra que con la formación se incrementan las consultas. Sin embargo, conviene aclarar que no todos los problemas éticos son tributarios de una consulta directa al CEA. Hay una escalera ascendente en la complejidad según la cual una buena parte de los problemas éticos se resuelven, en un primer 
escalón, con reflexión y deliberación personal a la cabecera del paciente. En un segundo escalón, encontraremos problemas éticos más complejos cuya resolución se puede alcanzar deliberando en el equipo o consultando con un colega. Y ya, en un tercer escalón, se situarán los dilemas más complejos que no se han resuelto en los escalones previos y requieren el asesoramiento de un CEA. Respetar el orden de esta escala de tres peldaños evita que, en el pleno de un CEA, se invierta tiempo ante problemas de "primer escalón" que se podrían resolver con la ayuda de quien ejerza funciones de "consultor". En cualquier caso, consideramos muy importante que los consultores informen al CEA de los casos atendidos y su resolución fuera del pleno de CEA.

Naturaleza del asesoramiento. Explicamos una y otra vez que el CEA es un órgano de naturaleza consultiva que no suplanta la responsabilidad de los profesionales, porque además así lo establecen la normativa de su constitución. Sin embargo, en el modo de realizar las consultas de casos problemáticos, con cierta frecuencia se pide que el CEA dicte sentencia, esperando una resolución para aplicarla al caso. Esto se contradice con la teoría de que el CEA no debe suplantar la responsabilidad de los facultativos, o de los propios pacientes, que tienen la responsabilidad de tomar decisiones. Es decir, a veces se recurre al comité como si fuera un tribunal cuyo veredicto servirá para descargarse de la responsabilidad en un caso conflictivo, lo cual no debería suceder.

Un modo de paliar el riesgo de este traspaso de responsabilidades, que va en contra de la naturaleza consultiva del CEA, sería advertir con claridad, a quien consulta, de que no puede descargar su responsabilidad en las recomendaciones que reciba del CEA. Este, en sentido estricto, lo que hace es ayudar al consultante a deliberar.

Consideramos esta cuestión sobre la naturaleza de la consulta como sujeta a debate, porque no es sencilla de gestionar, ya que quien recibe el informe puede dar a conocer su contenido. De modo que un informe incluso puede llegar a sede judicial. En cualquier caso, el CAE debería tener muy presente este aspecto para procurar, en la medida de lo posible, no hacer 
recomendaciones cerradas, evitando de este modo suplantar la responsabilidad de los profesionales. Es todo un arte elaborar informes con estas características, pero se debe intentar mediante revisiones de redacción exigentes realizadas por varios miembros del CEA.

Dedicación profesional. Este es un aspecto crucial. Hemos de aceptar que los CEA funcionan gracias a altas dosis de motivación y así seguirá siendo. Pero esto también ocurre en muchas otras tareas y funciones de los servicios de un hospital o de los centros de salud. Los espacios de excelencia se logran gracias al motor de la ética de las personas ${ }^{11}$.

Sin embargo, es necesario un mínimo de estructura, de recursos y de reconocimiento, porque las personas no son de acero y no se pueden exigir voluntarismos extremos durante un tiempo que deja de ser razonable. En un CEA debe haber diferentes niveles de responsabilidad y dedicación. Es necesario un núcleo sólido de dos o tres miembros que deben disponer de unas horas de dedicación, más allá de los tiempos de reunión o de dedicación a la elaboración puntual de un informe. Por otro lado, es imprescindible disponer de recursos de formación, en condiciones equivalentes a cualquier otra comisión o grupo de trabajo.

A un CEA no se le hace ningún favor cuando se le dan recursos de tiempo o de formación. Es decisivo que los centros sanitarios tengan gerentes con capacidad de liderazgo y sensibilidad para entender la trascendencia de lo que es un CEA y su potencialidad para dinamizar la vida de una institución, respetando al mismo tiempo su independencia.

\section{Rogelio Altisent \\ Teresa Fernández-Letamendi

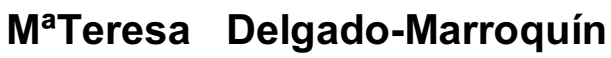


Una nueva vitalidad para el futuro de los comités de ética asistencial

Altisent, R; Fernández-Letamendi,T. y Delgado-Marroquín, MªT

$\underline{\text { http://doi.org/10.30860/0057 }}$

\section{BIBLIOGRAFIA}

1. Abel F. Comités de Ética Asistencial. An Sist Sanit Navar, 2006; 29 (S.3):75-83.

Disponible en (verificado abril 2019): http://scielo.isciii.es/pdf/asisna/v29s3/original7.pdf

2. Ribas-Ribas S. Estudio observacional sobre los comités de ética asistencial en Catalunya: el estudio CEA-CAT (1). Estructura y funcionamiento. Med Clin (Barc) 2006; 126(2): 60-66. Disponible en (verificado abril 2019):

http://si.easp.es/eticaysalud/sites/default/files/Ribas R Competencias CEAs Medicin a\%20Clinica.pdf

3. Ogando Diaz B., García Pérez C. Necesidades de formación en bioética en la Comunidad de Madrid. Aten Primaria 2005; 35:240-245. Disponible en (verificado abril 2019): doi:10.1157/13072788

4. Altisent R., Buil B., Delgado-Marroquín M.T. Are Ethics Committees in Tune with the "Epidemiology of Ethical Issues"? American Journal of Bioethics 2012; 12: 27-29. Disponible en (verificado abril 2019): http://dx.doi.org/10.1080/15265161.2012.719270

5. Baker R., Pearlman R., Taylor H., Kipnis K. Report and Recommendations of the American Society for Bioethics and Humanities. Advisory Committee on Ethics Standards, 2006. Citado en: Ravitsky V., Fiester A., Caplan A.L. The Penn Center Guide to Bioethics. New York: Springer Publishing Company, 2009. Disponible en (verificado abril 2019):

http://www.asbh.org/membership/protected/pdfs/acesrprt.pdf

6. Tarzian A J and the ASBH Core Competencies Update Task Force. Health care ethics consultation: An update on core competencies and emerging standards. American Journal of Bioethics 2013; 13:3-13. Disponible en (verificado abril 2019):

http://dx.doi.org/10.1080/15265161.2015.1021966

7. Hernando $P$ (coordinador). Comité de Bioética de Cataluña. La calidad en los comités de ética asistencial. Disponible en (verificado abril 2019):

http://canalsalut.gencat.cat/web/.content/ Sistema de salut/CBC/recursos/documents tematica/calidad comites etica asistencial.pdf

8. Fernández Letamendi T. Modelo de evaluación de la actividad de los comités de ética asistencial en Aragón (Tesis Doctoral, 2016), Disponible en (verificado abril 2019):

http://zaguan.unizar.es/record/48099/files/TESIS-2016-070.pdf

9. DuVal G., Clarridge B., Gensler G., Danis M. A National Survey of U.S. Internist. Experiences with Ethical Dilemmas and Ethics Consultation. Journal of General Internal Medicine, 2004; 19: 251-258. Disponible en (verificado abril 2019):

https://www.ncbi.nIm.nih.gov/pmc/articles/PMC1492156/pdf/jgi 21238.pdf

10. Beca J P. Consultores de ética clínica: razones, ventajas y limitaciones. Bioética\&Debat 2008; 14:1-5. Disponible en (verificado abril 2019):

http://medicina.udd.cl/centro-bioetica/files/2010/10/consultores.pdf 
Una nueva vitalidad para el futuro de los comités de ética asistencial

http://doi.org/10.30860/0057

Altisent, R; Fernández-Letamendi,T. y Delgado-Marroquín, MªT

11. Altisent R. Ética, Deontología y Derecho: lógicas diferentes en una misma dirección. Aten Primaria 2007; 39:225-226. Disponible en (verificado abril 2019):

https://www.elsevier.es/es-revista-atencion-primaria-27-pdf-13101794

\section{Cómo citar este artículo:}

Altisent, R.; Fernández-Letamendi, T.; Delgado-Marroquín, $\mathrm{M}^{\mathrm{a} T}$. "Una nueva vitalidad para el futuro de los comités de Ética asistencial" Folia Humanística, 2019 (13): 19-33. Doi: http://doi.org/10.30860/0057

(C) 2019 Todos los derechos reservados a la Revista Folia Humanística de la Fundación Letamendi Forns. This is an open access article. 